\title{
On approximating the modified Bessel function of the second kind
}

\author{
Zhen-Hang Yang ${ }^{1,2}$ and Yu-Ming Chu ${ }^{1 *}$
}

${ }^{*}$ Correspondence:

chuyuming2005@126.com

'School of Mathematics and Computation Sciences, Hunan City University, Yiyang, 413000, China Full list of author information is available at the end of the article

\section{Abstract}

In the article, we prove that the double inequalities

$$
\frac{\sqrt{\pi} e^{-x}}{\sqrt{2(x+a)}}<K_{0}(x)<\frac{\sqrt{\pi} e^{-x}}{\sqrt{2(x+b)}}, \quad 1+\frac{1}{2(x+a)}<\frac{K_{1}(x)}{K_{0}(x)}<1+\frac{1}{2(x+b)}
$$

hold for all $x>0$ if and only if $a \geq 1 / 4$ and $b=0$ if $a, b \in[0, \infty)$, where $K_{v}(x)$ is the modified Bessel function of the second kind. As applications, we provide bounds for $K_{n+1}(x) / K_{n}(x)$ with $n \in \mathbb{N}$ and present the necessary and sufficient condition such that the function $x \mapsto \sqrt{x+p} e^{x} K_{0}(x)$ is strictly increasing (decreasing) on $(0, \infty)$.

MSC: $33 \mathrm{~B} 10 ; 26 \mathrm{~A} 48$

Keywords: modified Bessel function; gamma function; monotonicity

\section{Introduction}

The modified Bessel function of the first kind $I_{v}(x)$ is a particular solution of the secondorder differential equation

$$
x^{2} y^{\prime \prime}(x)+x y^{\prime}(x)-\left(x^{2}+v^{2}\right) y(x)=0,
$$

and it can be expressed by the infinite series

$$
I_{\nu}(x)=\sum_{n=0}^{\infty} \frac{1}{n ! \Gamma(v+n+1)}\left(\frac{x}{2}\right)^{2 n+v} .
$$

While the modified Bessel function of the second kind $K_{v}(x)$ is defined by

$$
K_{v}(x)=\frac{\pi\left(I_{-v}(x)-I_{v}(x)\right)}{2 \sin (\pi v)},
$$

where the right-hand side of the identity of (1.1) is the limiting value in case $v$ is an integer.

The following integral representation formula and asymptotic formulas for the modified Bessel function of the second kind $K_{v}(x)$ can be found in the literature [1], 9.6.24, 9.6.8, 9.6.9, 9.7.2:

$$
K_{v}(x)=\int_{0}^{\infty} e^{-x \cosh (t)} \cosh (v t) d t \quad(x>0)
$$

(c) The Author(s) 2017. This article is distributed under the terms of the Creative Commons Attribution 4.0 International License (http://creativecommons.org/licenses/by/4.0/), which permits unrestricted use, distribution, and reproduction in any medium, provided you give appropriate credit to the original author(s) and the source, provide a link to the Creative Commons license, and indicate if changes were made. 


$$
\begin{aligned}
& K_{0}(x) \sim-\log x \quad(x \rightarrow 0), \\
& K_{v}(x) \sim \frac{1}{2} \Gamma(v)\left(\frac{x}{2}\right)^{-v} \quad(v>0, x \rightarrow 0), \\
& K_{v}(x) \sim \sqrt{\frac{\pi}{2 x}} e^{-x}\left[1+\frac{4 v^{2}-1}{8 x}+\frac{\left(4 v^{2}-1\right)\left(4 v^{2}-9\right)}{2 !(8 x)^{2}}+\cdots\right] \quad(x \rightarrow \infty) .
\end{aligned}
$$

From (1.2) we clearly see that

$$
\begin{aligned}
& K_{0}(x)=\int_{0}^{\infty} e^{-x \cosh (t)} d t=\int_{1}^{\infty} \frac{e^{-x t}}{\sqrt{t^{2}-1}} d t, \\
& K_{0}^{\prime}(x)=-\int_{1}^{\infty} \frac{t e^{-x t}}{\sqrt{t^{2}-1}} d t=-K_{1}(x) .
\end{aligned}
$$

Recently, the bounds for the modified Bessel function of the second kind $K_{v}(x)$ have attracted the attention of many researchers. Luke [2] proved that the double inequality

$$
\frac{8 \sqrt{x}}{8 x+1}<\sqrt{\frac{2}{\pi}} e^{x} K_{0}(x)<\frac{16 x+7}{(16 x+9) \sqrt{x}}
$$

holds for all $x>0$.

Gaunt [3] proved that the double inequality

$$
\frac{1}{\sqrt{x+\frac{1}{2}}}<\frac{\Gamma\left(x+\frac{1}{2}\right)}{\Gamma(x+1)}<\sqrt{\frac{2}{\pi}} e^{x} K_{0}(x)<\frac{1}{\sqrt{x}}
$$

takes place for all $x>0$, where $\Gamma(x)=\int_{0}^{\infty} t^{x-1} e^{-t} d x$ is the classical gamma function.

In [4], Segura proved that the double inequality

$$
\frac{v+\sqrt{x^{2}+v^{2}}}{x}<\frac{K_{v+1}(x)}{K_{v}(x)}<\frac{v+\frac{1}{2}+\sqrt{x^{2}+\left(v+\frac{1}{2}\right)^{2}}}{x}
$$

holds for all $x>0$ and $v \geq 0$.

Bordelon and Ross [5] and Paris [6] provided the inequality

$$
\frac{K_{v}(x)}{K_{v}(y)}>e^{y-x}\left(\frac{x}{y}\right)^{v}
$$

for all $v>-1 / 2$ and $y>x>0$.

Laforgia [7] established the inequality

$$
\frac{K_{v}(x)}{K_{v}(y)}>e^{y-x}\left(\frac{x}{y}\right)^{-v}
$$

for all $y>x>0$ if $v \in(0,1 / 2)$, and inequality (1.12) is reversed if $v \in(1 / 2, \infty)$.

Baricz [8] presented the inequality

$$
\frac{K_{v}(x)}{K_{v}(y)}>e^{y-x}\left(\frac{x}{y}\right)^{-1 / 2}
$$

for all $y>x>0$ and $v \in(-\infty,-1 / 2) \cup(1 / 2, \infty)$. 
Motivated by inequality (1.9), in the article, we prove that the double inequality

$$
\frac{\sqrt{\pi} e^{-x}}{\sqrt{2(x+a)}}<K_{0}(x)<\frac{\sqrt{\pi} e^{-x}}{\sqrt{2(x+b)}}
$$

holds for all $x>0$ if and only if $a \geq 1 / 4$ and $b=0$ if $a, b \in[0, \infty)$. As applications, we provide bounds for $K_{n+1}(x) / K_{n}(x)$ with $n \in \mathbb{N}$ and present the necessary and sufficient condition such that the function $x \mapsto \sqrt{x+p} e^{x} K_{0}(x)$ is strictly increasing (decreasing) on $(0, \infty)$.

\section{Lemmas}

In order to prove our main results, we need two lemmas which we present in this section.

Lemma 2.1 (See [9]) Let $-\infty \leq a<b \leq \infty, f, g:[a, b] \rightarrow \mathbb{R}$ be continuous on $[a, b]$ and differentiable on $(a, b)$, and $g^{\prime}(x) \neq 0$ on $(a, b)$. If $f^{\prime}(x) / g^{\prime}(x)$ is increasing (decreasing) on $(a, b)$, then so are the functions

$$
\frac{f(x)-f(a)}{g(x)-g(a)}, \quad \frac{f(x)-f(b)}{g(x)-g(b)}
$$

If $f^{\prime}(x) / g^{\prime}(x)$ is strictly monotone, then the monotonicity in the conclusion is also strict.

Lemma 2.2 The function

$$
x \mapsto f(x)=\frac{K_{0}(x)}{2\left[K_{1}(x)-K_{0}(x)\right]}-x
$$

is strictly increasing from $(0, \infty)$ onto $(0,1 / 4)$.

Proof Let $\omega(t)=\sqrt{(t-1) /(t+1)}$. Then it follows from (1.6), (1.7) and (2.1) that

$$
\begin{aligned}
& K_{1}(x)-K_{0}(x)=\int_{1}^{\infty} \omega(t) e^{-x t} d t \\
& \begin{aligned}
x\left[K_{1}(x)-K_{0}(x)\right] & =-\int_{1}^{\infty} \omega(t) d\left(e^{-x t}\right) \\
& =\left.\omega(t) e^{-x t}\right|_{t=\infty} ^{t=1}+\int_{1}^{\infty} \omega^{\prime}(t) e^{-x t} d t \\
& =\int_{1}^{\infty} \frac{t-1}{\left(t^{2}-1\right)^{3 / 2}} e^{-x t} d t
\end{aligned} \\
& \begin{aligned}
K_{0}(x)-2 x\left[K_{1}(x)-K_{0}(x)\right]=\int_{1}^{\infty} \frac{\omega(t)}{t+1} e^{-x t} d t \\
f(x)=\frac{K_{0}(x)-2 x\left[K_{1}(x)-K_{0}(x)\right]}{2\left[K_{1}(x)-K_{0}(x)\right]}=\frac{\int_{1}^{\infty} \frac{\omega(t)}{t+1} e^{-x t} d t}{2 \int_{1}^{\infty} \omega(t) e^{-x t} d t}, \\
f^{\prime}(x)=\frac{-\int_{1}^{\infty} \frac{t \omega(t)}{t+1} e^{-x t} d t \int_{1}^{\infty} \omega(t) e^{-x t} d t+\int_{1}^{\infty} \frac{\omega(t)}{t+1} e^{-x t} d t \int_{1}^{\infty} t \omega(t) e^{-x t} d t}{2\left(\int_{1}^{\infty} \omega(t) e^{-x t} d t\right)^{2}} \\
=\frac{\int_{1}^{\infty}\left(\int_{1}^{\infty} \frac{s-t}{t+1} \omega(t) \omega(s) e^{-x(s+t)} d t\right) d s}{2\left(\int_{1}^{\infty} \omega(t) e^{-x t} d t\right)^{2}}=\frac{\int_{1}^{\infty}\left(\int_{1}^{\infty} \frac{t-s}{s+1} \omega(s) \omega(t) e^{-x(t+s)} d s\right) d t}{2\left(\int_{1}^{\infty} \omega(t) e^{-x t} d t\right)^{2}}
\end{aligned}
\end{aligned}
$$




$$
\begin{aligned}
& =\frac{\int_{1}^{\infty}\left(\int_{1}^{\infty} \frac{s-t}{t+1} \omega(t) \omega(s) e^{-x(s+t)} d t\right) d s+\int_{1}^{\infty}\left(\int_{1}^{\infty} \frac{t-s}{s+1} \omega(s) \omega(t) e^{-x(t+s)} d s\right) d t}{4\left(\int_{1}^{\infty} \omega(t) e^{-x t} d t\right)^{2}} \\
& =\frac{\int_{1}^{\infty} \int_{1}^{\infty} \frac{(s-t)^{2}}{(t+1)(s+1)} \omega(t) \omega(s) e^{-x(t+s)} d t d s}{4\left(\int_{1}^{\infty} \omega(t) e^{-x t} d t\right)^{2}}>0
\end{aligned}
$$

for all $x>0$.

Note that (1.3)-(1.5) and (2.1) lead to

$$
\begin{aligned}
& \lim _{x \rightarrow 0} x K_{0}(x)=0, \quad \lim _{x \rightarrow 0} x K_{1}(x)=1, \\
& \lim _{x \rightarrow 0} f(x)=\lim _{x \rightarrow 0}\left[\frac{x K_{0}(x)}{2\left(x K_{1}(x)-x K_{0}(x)\right)}-x\right]=0, \\
& \lim _{x \rightarrow \infty} f(x)=\lim _{x \rightarrow \infty}\left[\frac{K_{0}(x)-2 x\left(K_{1}(x)-K_{0}(x)\right)}{2\left(K_{1}(x)-K_{0}(x)\right)}\right]=\lim _{x \rightarrow \infty} \frac{\frac{1}{4 x}+o\left(\frac{1}{x}\right)}{\frac{1}{x}+o\left(\frac{1}{x}\right)}=\frac{1}{4} .
\end{aligned}
$$

Therefore, Lemma 2.2 follows easily from (2.2)-(2.4).

\section{Main results}

Theorem 3.1 Let $a, b \geq 0$. Then the double inequality

$$
\frac{1}{\sqrt{x+a}}<\sqrt{\frac{2}{\pi}} e^{x} K_{0}(x)<\frac{1}{\sqrt{x+b}}
$$

holds for all $x>0$ if and only if $a \geq 1 / 4$ and $b=0$.

Proof Let $x>0, f(x)$ be defined by Lemma 2.2, and $f_{1}(x), f_{2}(x)$ and $F(x)$ be respectively defined by

$$
f_{1}(x)=\frac{\pi}{2}-x e^{2 x} K_{0}^{2}(x), \quad f_{2}(x)=e^{2 x} K_{0}^{2}(x)
$$

and

$$
F(x)=\frac{\frac{\pi}{2}-x e^{2 x} K_{0}^{2}(x)}{e^{2 x} K_{0}^{2}(x)}=\frac{f_{1}(x)}{f_{2}(x)} .
$$

Then from (1.5), (1.7) and (3.1) we clearly see that

$$
\begin{aligned}
& \lim _{x \rightarrow \infty} f_{1}(x)=\lim _{x \rightarrow \infty} f_{2}(x)=0, \\
& \frac{f_{1}^{\prime}(x)}{f_{2}^{\prime}(x)}=\frac{-e^{2 x} K_{0}^{2}(x)+2 x e^{2 x} K_{0}(x)\left[K_{1}(x)-K_{0}(x)\right]}{-2 e^{2 x} K_{0}(x)\left[K_{1}(x)-K_{0}(x)\right]}=f(x) .
\end{aligned}
$$

It follows from (3.2)-(3.4), Lemmas 2.1 and 2.2 together with L'Hôpital's rule that the function $F(x)$ is strictly increasing on $(0, \infty)$ and

$$
\lim _{x \rightarrow \infty} F(x)=\frac{1}{4}
$$


Note that (1.3) and (3.2) lead to

$$
\lim _{x \rightarrow 0} F(x)=\lim _{x \rightarrow 0}\left[\frac{\pi}{2 e^{2 x} K_{0}^{2}(x)}-x\right]=0 .
$$

Therefore, Theorem 3.1 follows easily from (3.2), (3.5), (3.6) and the monotonicity of $F(x)$.

Remark 3.2 From Lemma 2.2 we clearly see that the double inequality

$$
p<\frac{K_{0}(x)}{2\left[K_{1}(x)-K_{0}(x)\right]}-x<q
$$

holds for all $x>0$ if and only if $p \leq 0$ and $q \geq 1 / 4$.

From (1.7) and Remark 3.2 we get Corollary 3.3 immediately.

Corollary 3.3 Let $p, q \geq 0$. Then the double inequalities

$$
1+\frac{1}{2(x+p)}<\frac{K_{1}(x)}{K_{0}(x)}<1+\frac{1}{2(x+q)}
$$

and

$$
\left[\log \left(e^{x} \sqrt{x+p}\right)\right]^{\prime}<-\left[\log K_{0}(x)\right]^{\prime}<\left[\log \left(e^{x} \sqrt{x+q}\right)\right]^{\prime}
$$

hold for all $x>0$ if and only if $p \geq 1 / 4$ and $q=0$.

Remark 3.4 Let $p \geq 0$. Then from inequality (3.7) we know that the function $x \mapsto$ $\sqrt{x+p} e^{x} K_{0}(x)$ is strictly increasing (decreasing) on $(0, \infty)$ if and only if $p=0(p \geq 1 / 4)$. We clearly see that the bounds for $K_{1}(x) / K_{0}(x)$ given in Corollary 3.3 are better than the bounds given in (1.10) for $v=0$.

From (1.3), (1.5) and Remark 3.4 we get Corollary 3.5 immediately.

Corollary 3.5 The double inequality

$$
\sqrt{\frac{\pi}{2}}=\lim _{x \rightarrow \infty}\left[\sqrt{x+p} e^{x} K_{0}(x)\right]<\left[\sqrt{x+p} e^{x} K_{0}(x)\right]<\lim _{x \rightarrow 0}\left[\sqrt{x+p} e^{x} K_{0}(x)\right]=\infty
$$

holds for all $x>0$ if $p \geq 1 / 4$, and inequality (3.8) is reversed if $p=0$.

Remark 3.4 also leads to Corollary 3.6.

Corollary 3.6 Let $p, q \geq 0$. Then the double inequality

$$
\sqrt{\frac{y+p}{x+p}} e^{y-x}<\frac{K_{0}(x)}{K_{0}(y)}<\sqrt{\frac{y+q}{x+q}} e^{y-x}
$$

holds for all $0<x<y$ if and only if $p \geq 1 / 4$ and $q=0$. 
Remark 3.7 We clearly see that the lower bound for $K_{0}(x) / K_{0}(y)$ in Corollary 3.6 is better than the bounds given in (1.11) and (1.12) for $v=0$.

Remark 3.8 From the inequality

$$
\frac{\Gamma\left(x+\frac{1}{2}\right)}{\Gamma(x+1)}<\frac{1}{\sqrt{x+\frac{1}{4}}}
$$

given in [10], (2.20), and the fact that

$$
\frac{1}{\sqrt{x+\frac{1}{4}}}>\frac{8 \sqrt{x}}{8 x+1}
$$

for all $x>0$ we clearly see that the lower bound given in Theorem 3.1 for $\sqrt{2 / \pi} e^{x} K_{0}(x)$ is better than that given in (1.8) and (1.9). But the upper bound given in Theorem 3.1 is weaker than that given in (1.8).

Remark 3.9 From Theorem 3.1 and Corollary 3.3 we clearly see that there exist $\theta_{1}=$ $\theta_{1}(x) \in(0,1 / 4)$ and $\theta_{2}=\theta_{2}(x) \in(0,1 / 4)$ such that

$$
K_{0}(x)=\sqrt{\frac{\pi}{2\left(x+\theta_{1}\right)}} e^{-x}, \quad K_{1}(x)=\left[1+\frac{1}{2\left(x+\theta_{2}\right)}\right] \sqrt{\frac{\pi}{2\left(x+\theta_{1}\right)}} e^{-x}
$$

for all $x>0$.

Theorem 3.10 Let $x>0, n \in \mathbb{N}, R_{n}(x)=K_{n+1}(x) / K_{n}(x), u_{0}(x)=1+1 /(2 x), v_{0}(x)=1+1 /(2 x+$ $1 / 2)$, and $u_{n}(x)$ and $v_{n}(x)$ be defined by

$$
u_{n}(x)=\frac{1}{v_{n-1}(x)}+\frac{2 n}{x}, \quad v_{n}(x)=\frac{1}{u_{n-1}(x)}+\frac{2 n}{x} \quad(n \geq 1) .
$$

Then the double inequality

$$
v_{n}(x)<R_{n}(x)=\frac{K_{n+1}(x)}{K_{n}(x)}<u_{n}(x)
$$

holds for all $x>0$ and $n \in \mathbb{N}$.

Proof We use mathematical induction to prove inequality (3.10). From Corollary 3.3 we clearly see that inequality (3.10) holds for all $x>0$ and $n=0$.

Suppose that inequality (3.10) holds for $n=k-1(k \geq 1)$, that is,

$$
v_{k-1}(x)<R_{k-1}(x)<u_{k-1}(x) .
$$

Then it follows from (3.9) and (3.11) together with the formula

$$
\frac{K_{v}^{\prime}(x)}{K_{v}(x)}=-\frac{K_{v-1}(x)}{K_{v}(x)}-\frac{v}{x}=-\frac{K_{v+1}(x)}{K_{v}(x)}+\frac{v}{x}
$$


given in [11] that

$$
\begin{aligned}
& R_{k}(x)=\frac{1}{R_{k-1}(x)}+\frac{2 k}{x}, \\
& \nu_{k}(x)=\frac{1}{u_{k-1}(x)}+\frac{2 k}{x}<R_{k}(x)<\frac{1}{v_{k-1}(x)}+\frac{2 k}{x}=u_{k}(x) .
\end{aligned}
$$

Inequality (3.12) implies that inequality (3.10) holds for $n=k$, and the proof of Theorem 3.10 is completed.

Remark 3.11 Let $n=1,2,3$. Then Theorem 3.10 leads to

$$
\begin{aligned}
& \frac{2(x+1)^{2}}{x(2 x+1)}<\frac{K_{2}(x)}{K_{1}(x)}<\frac{4 x^{2}+9 x+6}{x(4 x+3)} \\
& \frac{4 x^{3}+19 x^{2}+36 x+24}{x\left(4 x^{2}+9 x+6\right)}<\frac{K_{3}(x)}{K_{2}(x)}<\frac{2 x^{3}+9 x^{2}+16 x+8}{2 x(x+1)^{2}} \\
& \frac{2\left(x^{4}+8 x^{3}+28 x^{2}+48 x+24\right)}{x\left(2 x^{3}+9 x^{2}+16 x+8\right)}<\frac{K_{4}(x)}{K_{3}(x)}<\frac{4 x^{4}+33 x^{3}+120 x^{2}+216 x+144}{x\left(4 x^{3}+19 x^{2}+36 x+24\right)}
\end{aligned}
$$

for all $x>0$.

Remark 3.12 It is not difficult to verify that

$$
\begin{aligned}
& \frac{2(x+1)^{2}}{x(2 x+1)}>\frac{1+\sqrt{x^{2}+1}}{x}, \quad \frac{4 x^{2}+9 x+6}{x(4 x+3)}<\frac{\frac{3}{2}+\sqrt{x^{2}+\frac{9}{4}}}{x}, \\
& \frac{4 x^{3}+19 x^{2}+36 x+24}{x\left(4 x^{2}+9 x+6\right)}>\frac{2+\sqrt{x^{2}+4}}{x}, \quad \frac{2 x^{3}+9 x^{2}+16 x+8}{2 x(x+1)^{2}}<\frac{\frac{5}{2}+\sqrt{x^{2}+\frac{25}{4}}}{x}, \\
& \frac{2\left(x^{4}+8 x^{3}+28 x^{2}+48 x+24\right)}{x\left(2 x^{3}+9 x^{2}+16 x+8\right)}>\frac{3+\sqrt{x^{2}+9}}{x} \\
& \frac{4 x^{4}+33 x^{3}+120 x^{2}+216 x+144}{x\left(4 x^{3}+19 x^{2}+36 x+24\right)}<\frac{\frac{7}{2}+\sqrt{x^{2}+\frac{49}{4}}}{x}
\end{aligned}
$$

for $x>0$. Therefore, the bounds given in Remark 3.11 are better than the bounds given in (1.10) for $v=1,2,3$.

\section{Competing interests}

The authors declare that they have no competing interests.

\section{Authors' contributions}

All authors contributed equally to the writing of this paper. All authors read and approved the final manuscript.

\section{Author details}

${ }^{1}$ School of Mathematics and Computation Sciences, Hunan City University, Yiyang, 413000, China. ${ }^{2}$ Customer Service Center, State Grid Zhejiang Electric Power Research Institute, Hangzhou, 310009, China.

\section{Acknowledgements}

The research was supported by the Natural Science Foundation of China under Grants 61673169, 61374086, 11371125 and 11401191.

Received: 10 January 2017 Accepted: 8 February 2017 Published online: 13 February 2017 
References

1. Abramowitz, M, Stegun, IA: Handbook of Mathematical Functions with Formulas, Graphs, and Mathematical Tables. Dover, New York (1970)

2. Luke, YL: Inequalities for generalized hypergeometric functions. J. Approx. Theory 5, 41-65 (1972)

3. Gaunt, RE: Inequalities for modified Bessel functions and their integrals. J. Math. Anal. Appl. 420(1), 373-386 (2014)

4. Segura, J: Bounds for ratios of modified Bessel functions and associated Turán-type inequalities. J. Math. Anal. Appl. 374(2), 516-528 (2011)

5. Bordelon, DJ, Ross, DK: Problem 72-75, 'Inequalities for special functions'. SIAM Rev. 15, 665-670 (1973)

6. Paris, RB: An inequality for the Bessel function $J_{v}(v x)$. SIAM J. Math. Anal. 15(1), 203-205 (1984)

7. Laforgia, A: Bounds for modified Bessel functions. J. Comput. Appl. Math. 34(3), 263-267 (1991)

8. Baricz, Á: Bounds for modified Bessel functions of the first and second kinds. Proc. Edinb. Math. Soc. (2) 53(3), 575-599 (2010)

9. Anderson, GD, Vamanamurthy, MK, Vuorinen, M: Monotonicity rules in calculus. Am. Math. Mon. 113(9), 805-816 (2006)

10. Qi, F: Bounds for the ratio of two gamma functions. J. Inequal. Appl. 2010, Article ID 493085 (2010)

11. Watson, GN: A Treatise on the Theory of Bessel Functions. Cambridge University Press, Cambridge (1995)

\section{Submit your manuscript to a SpringerOpen ${ }^{\circ}$ journal and benefit from:}

- Convenient online submission

- Rigorous peer review

- Immediate publication on acceptance

- Open access: articles freely available online

- High visibility within the field

- Retaining the copyright to your article 